\title{
An Optimal Inventory Pricing and Ordering Strategy Subject to Stock and Price Dependent Demand
}

\author{
A. Tsoularis \\ Business Rose Bowl \\ Leeds Metropolitan University \\ Portland Crescent, Leeds, LS1 3HB, UK \\ e-mail: a.tsoularis@leedsmet.ac.uk
}

\author{
J. Wallace \\ School of Management \\ Bradford University \\ Emm Lane, Bradford, BD9 4JL, UK \\ e-mail: j.wallace1@bradford.ac.uk
}

Received: July 12, 2019. Revised: September 30, 2021. Accepted: October 22, 2021. Published: November 23, 2021.

\begin{abstract}
This article considers the deterministic optimal control problem of profit maximization for inventory replenished at a variable rate and depleted by demand which is assumed to vary with price and stock availability. Optimal policies for the inventor, product order rate and price are derived using the maximum principle. Bounds on the maximum price possible are also derived.
\end{abstract}

Keywords-Optimal Control, Hamiltonian, costate variable, maximum principle, bang-bang control

\section{INTRODUCTION}

The main premise of this article is that the demand for a particular product may increase linearly as more stock on display becomes available. This idea was first suggested by Wolfe [1]. Optimal ordering strategies adopting the Economic Order Quantity $(E O Q)$ were addressed by Baker and Urban [2], Urban [3-4] and Gerchak and Wang [5]. In addition to the inventory we introduce demand dependence on the price of the product in a convex manner. A linear price dependence was investigated by Jørgensen and Kort [6]. Our objective is to maximize the net profit from selling the product incorporating holding and ordering costs over a finite horizon. A similar objective in an infinite horizon setting was used by Khmelnitsky and Gerchak [7] who excluded demand price dependence.

\section{THE OPTIMAL CONTROL MODEL}

\section{A. Model definition}

The demand for an item is a function of the inventory in stock at time $t, x(t)$, and the price, $p$. Specifically, the demand rate, $d(x, p)$, is given by

$$
d(x, p)=a x(b-p)^{2}
$$

where $a$ is a suitable growth parameter and $b$ is the maximum product price possible, $p \leq b$. The demand rate will increase as $x$ increases, $\frac{\partial d}{\partial x}>0$, and will decline as $p$ increases, $\frac{\partial d}{\partial p}<0$, with demand ceasing to grow altogether when either $x=0$ or $p=b, d(0, p)=d(x, b)=0$. As price increases, the demand rate will decline with the available stock, $\frac{\partial^{2} d}{\partial p \partial x}<0$

The inventory evolves according to the differential equation

$$
\dot{x}=u-d(x, p)=u-a x(b-p)^{2}
$$

where $u$ is the order (replenishment) rate, $0 \leq u \leq U$ and $0 \leq p \leq b$ and $x(0)=x_{0}$.

The objective is to select an order and pricing policy so as to maximize the net profit over a horizon $T$ :

$$
\max _{u, p}\left(\int_{0}^{T}(p d(x, p)-h x-c u) d t\right)
$$

where $c$ is the unit order cost and $h$ is the unit holding cost, $c<b$.

\section{B. The costate variable and the Hamiltonian function}

The Hamiltonian is defined as

$$
\begin{aligned}
& \mathcal{H}(x, u, p, \lambda)= \\
& \operatorname{pax}(b-p)^{2}-h x-c u+\lambda\left(u-a x(b-p)^{2}\right)
\end{aligned}
$$

where $\lambda$ is the costate variable measuring the shadow price of the inventory variable, $x$ (Chiang [8]). The maximum principle conditions are

$$
\frac{\partial \mathcal{H}}{\partial u}=0 \text { and } \frac{\partial \mathcal{H}}{\partial p}=0 \text { optimality conditions }
$$




$$
\begin{gathered}
\dot{\lambda}=-\frac{\partial \mathcal{H}}{\partial x} \text { trajectory of } \lambda(t) \\
\lambda(T)=0 \text { transversality condition }
\end{gathered}
$$

The maximum principle conditions (5) are necessary but not sufficient for maximizing the Hamiltonian. Arrow's sufficiency theorem must be applied to the Hamiltonian to ensure sufficiency by proving the concavity of the Hamiltonian (Kamien and Schwartz [9]).

\section{Solution to the optimal control problem}

The optimality condition, $\frac{\partial \mathcal{H}}{\partial u}=0$, cannot be strictly applied because the Hamiltonian is linear in $u$. Since $\frac{\partial \mathcal{H}}{\partial u}=\lambda-c$, this is a case of bang-bang control insofar as the optimal ordering policy is concerned. We have then for the optimal ordering policy, $u^{*}$ :

$$
u^{*}=\left\{\begin{array}{c}
0 \text { if } \lambda<c \\
\text { indetermirateif } \lambda=c \\
U \text { if } \lambda>c
\end{array}\right.
$$

The second optimality condition, $\frac{\partial \not{H}}{\partial p}=0$, yields the optimal pricing policy

$$
p^{*}=\frac{b+2 \lambda}{3}
$$

From (6) we have

$$
\dot{\lambda}=h-a(p-\lambda)(b-p)^{2}
$$

The price, $p$, must be by definition non-negative. This leads to the identification of four distinct regions $A, B, C$, and $D$ :

\section{Region $A$}

$$
\begin{aligned}
& \lambda \leq-\frac{b}{2}, p^{*}=0, u^{*}=0 \\
& \dot{x}=-a b^{2} x, \dot{\lambda}=a b^{2} \lambda+h
\end{aligned}
$$

The Hamiltonian in this region is given by

$$
\mathcal{H}(x, \lambda)=-\left(h+\lambda a b^{2}\right) x
$$

which is linear in $x$ for a given $\lambda$ and automatically concave.

\section{$\underline{\text { Region } B}$}

$$
\begin{aligned}
& -\frac{b}{2}<\lambda \leq c, p^{*}=\frac{b+2 \lambda}{3}, u^{*}=0 \\
& \dot{x}=-\frac{4 a x}{9}(b-\lambda)^{2}, \dot{\lambda}=h-\frac{4 a}{27}(b-\lambda)^{3}
\end{aligned}
$$

Here the Hamiltonian is given by

$$
\begin{aligned}
& \mathcal{H}(x, u, p, \lambda)= \\
& \operatorname{pax}(b-p)^{2}-h x-\lambda a x(b-p)^{2}= \\
& \left(\frac{4 a}{27}(b-\lambda)^{3}-h\right) x
\end{aligned}
$$

which is linear in $x$ for a given $\lambda$ and automatically concave.

$\underline{\operatorname{Region} C}$

$$
\begin{aligned}
& c<\lambda \leq b, p^{*}=\frac{b+2 \lambda}{3}, u^{*}=U \\
& \dot{x}=U-\frac{4 a x}{9}(b-\lambda)^{2}, \dot{\lambda}=h-\frac{4 a}{27}(b-\lambda)^{3}
\end{aligned}
$$

Here the Hamiltonian is given by

$$
\begin{aligned}
& \mathcal{H}(x, u, p, \lambda)= \\
& \operatorname{pax}(b-p)^{2}-h x-c U+\lambda\left(U-a x(b-p)^{2}\right)= \\
& \left(\frac{4 a}{27} a(b-\lambda)^{3}-h\right) x+U(\lambda-c)
\end{aligned}
$$

which is also linear in $x$ for a given $\lambda$ and automatically concave.

$\underline{\operatorname{Region} D}$

$$
\lambda>b, p^{*}=b, u^{*}=U, \dot{x}=U, \dot{\lambda}=h
$$

The Hamiltonian is given by

$$
\mathcal{H}(x, u, p, \lambda)=h x+(\lambda-c) U
$$

again linear in $x$ for a given $\lambda$ and automatically concave.

If the shadow price is negative it is not profitable to replenish the stock $\left(u^{*}=0\right)$ and the price is set to $0\left(p^{*}=0\right)$ in order to get rid of the stock. If the shadow price reaches positive values but remains below the unit order cost, $c$, it is still not profitable to replenish but the price can be set at some low value to get rid of the remaining stock. Maximum order rates and variable pricing are applied when the shadow price exceeds the unit order cost but remains below the maximum possible price, $b$. As soon as the shadow price attains $b$, the price is set at its maximum, $b$, and maximum order rates are applied.

Region $D$ is ruled out as part of the optimal policy because the inventory and the shadow price grow linearly in time, both starting from positive values, hence the transversality condition 
(7) cannot be met. As such none of the other three regions are accessible from $D$.

Region $B$ must be the terminal region as it is the only one for which the transversality condition is met. The transitions $A \rightarrow C \rightarrow B$ and $C \rightarrow A \rightarrow B$ are both precluded by the continuity property of the costate variable. It remains to investigate whether region $B$ is accessible from the other two adjacent regions, $A$ and $C$.

\section{$A \rightarrow B$ :}

In region $A$, the solution to $\dot{\lambda}=h+a b^{2} \lambda$ is

$$
\lambda^{*}(t)=k_{1} e^{a b^{2} t}-\frac{h}{a b^{2}}
$$

where $k_{1}$ is the integration constant. If region $B$ is to be reached the costate variable must be constantly increasing in time as the inventory declines exponentially $\left(x(t) \sim e^{-a b^{2} t}\right)$ as no ordering is taking place. An increase is possible only if $k_{1}>0$. For the costate variable to increase, $\dot{\lambda}=h+a b^{2} \lambda>0$, or $\lambda(t) \geq-\frac{h}{a b^{2}}$. Since $\lambda(t) \leq-\frac{b}{2}$, the following condition must be met:

$$
b \leq\left(\frac{2 h}{a}\right)^{\frac{1}{3}}
$$

In other words, the maximum price, $b$, must be bounded from above to allow the shadow price to rise to $-\frac{b}{2}$.

$B \rightarrow B$ :

In this region $\dot{\lambda}=h-\frac{4 a}{27}(b-\lambda)^{3}$. The explicit solution to the equation with integration constant, $k_{2}$, is realised by separation of variables and subsequent partial fraction expansion of the integrand:

$$
\frac{1}{\left(4 a h^{2}\right)^{\frac{1}{3}}}\left(\int \frac{d z}{z-\theta}-\int \frac{z+2 \theta}{z^{2}+\theta z+\theta^{2}} d z\right)=t+k_{2}
$$

where $\theta=\left(\frac{27 h}{4 a}\right)^{\frac{1}{3}}$ and $z=b-\lambda$. Straightforward integration yields

$$
\begin{gathered}
\frac{1}{\left(4 a h^{2}\right)^{\frac{1}{3}}}\left(\ln \left(\frac{|z-\theta|}{\sqrt{z^{2}+\theta z+\theta^{2}}}\right)-\sqrt{3} \arctan \left(\frac{2 z+\theta}{\theta \sqrt{3}}\right)\right) \\
=t+k_{2}
\end{gathered}
$$

Applying boundary condition (7), we get the full solution in region $B$ :

$$
\begin{aligned}
& \frac{1}{\left(4 a h^{2}\right)^{\frac{1}{3}}}\left(\ln \left(\left|\frac{b-\theta}{z-\theta}\right| \sqrt{\frac{z^{2}+z \theta+\theta^{2}}{b^{2}+b \theta+\theta^{2}}}\right)\right) \\
& -\frac{\sqrt{3}}{\left(4 a h^{2}\right)^{\frac{1}{3}}} \arctan \left(\frac{\theta \sqrt{3}(b-z)}{2 \theta^{2}+2 b z+\theta(b+z)}\right)=T-t
\end{aligned}
$$

For negative values of the costate variable condition (12) ensures that the terminal value, $\lambda(T)=0$ will be reached. For positive values of the costate variable the following condition must be held

$$
b>c+\frac{3}{2}\left(\frac{2 h}{a}\right)^{\frac{1}{3}}
$$

$C \rightarrow B:$

The system of the state equations in region $C$

$$
\begin{aligned}
& \dot{x}=U-\frac{4 a x}{9}(b-\lambda)^{2} \\
& \dot{\lambda}=h-\frac{4 a}{27}(b-\lambda)^{3}
\end{aligned}
$$

possesses a unique equilibrium:

$$
\begin{aligned}
& \bar{x}=U\left(4 a h^{2}\right)^{-\frac{1}{3}} \\
& \bar{\lambda}=b-\frac{3}{2}\left(\frac{2 h}{a}\right)^{\frac{1}{3}}
\end{aligned}
$$

Throughout region $C, \lambda>c$, hence the following condition must be met for the saddle costate equilibrium value to exist:

$$
b>c+\frac{3}{2}\left(\frac{2 h}{a}\right)^{\frac{1}{3}}
$$

The Jacobian matrix possesses two real eigenvalues, equal of opposite sign, $\mu_{1}=\left(4 a h^{2}\right)^{\frac{1}{3}}, \mu_{2}=-\left(4 a h^{2}\right)^{\frac{1}{3}}$. The equilibrium is a saddle point, hence it is unstable. The perturbations $(\xi, \eta)$ from the equilibrium $(\bar{x}, \bar{\lambda})$ obey 


$$
\begin{aligned}
& \dot{\xi}=\mu_{2} \xi+\frac{2 U}{3}(4 a h)^{\frac{1}{3}} \eta \\
& \dot{\eta}=\mu_{1} \eta
\end{aligned}
$$

whence

$$
\begin{aligned}
& \xi(t)=\left(\xi_{0}-\frac{\eta_{0} U h^{-\frac{1}{3}}}{3}\right) e^{\mu_{2} t}+\frac{U h^{-\frac{1}{3}}}{3} \eta(t) \\
& \eta(t)=\eta_{0} e^{\mu_{1} t}
\end{aligned}
$$

The stable manifold is the $\eta=0$ axis and the unstable manifold is the straight line $\eta(t)=\frac{3 h^{\frac{1}{3}}}{U} \xi(t)$.

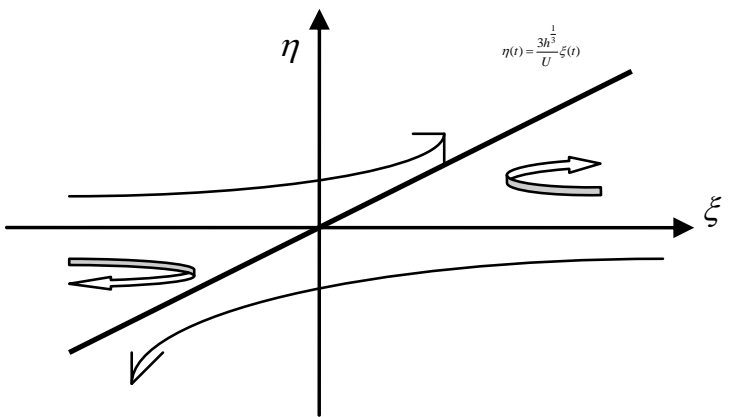

Figure 1. Saddle point $(\bar{x}, \bar{\lambda})$ is at the origin of the $(\xi, \eta)$ axes. When $\lambda>\bar{\lambda}, \dot{\lambda}>0$ and the costate variable keeps increasing. For the region $B$ to be accessible from region $C, \lambda$ must be decreasing, that is, $\dot{\lambda}<0$ or $\lambda<b-\left(\frac{27 h}{4 a}\right)^{\frac{1}{3}}$. But $\lambda=\frac{3 p-b}{2}$, hence $p<b-\left(\frac{2 h}{a}\right)^{\frac{1}{3}}$.The pricing policy is to set the price, $p$, by a margin of at least $\left(\frac{2 h}{a}\right)^{\frac{1}{3}}$ below the maximum price $b$.

\section{IMPLEMENTATION OF THE CONTROL POLICIES} $\underline{\text { Initial inventory starts in region } A}$

If the inventory falls in region $A$ and $b \leq\left(\frac{2 h}{a}\right)^{\frac{1}{3}}$, no ordering takes place $(u=0)$ and the price, $p$, is set to 0 to allow the inventory to decline exponentially whilst simultaneously the shadow price begins to rise as profit losses are reduced. At time, $t=\tau_{1}$, the inventory crosses over to region $B$, where the shadow price is $\lambda_{1}=-\frac{b}{2}$. $\tau_{1}$ can be explicitly calculated from (13) by setting the variable $z=b-\lambda=\frac{3 b}{2}$ and solving for $\tau_{1}$ :

$$
\begin{aligned}
& \tau_{1}=T-\frac{1}{\left(4 a h^{2}\right)^{\frac{1}{3}}}\left(\ln \left(\left|\frac{b-2 \theta}{3 b-2 \theta}\right| \sqrt{\frac{9 b^{2}+6 b \theta+4 \theta^{2}}{b^{2}+2 b \theta+4 \theta^{2}}}\right)\right) \\
& +\frac{\sqrt{3}}{\left(4 a h^{2}\right)^{\frac{1}{3}}} \arctan \left(-\frac{b \theta \sqrt{3}}{4 \theta^{2}+6 b^{2}+5 b \theta}\right)
\end{aligned}
$$

$\underline{\text { Initial inventory in region } B}$

If the maximum price, $b$, obeys the condition (12) then the costate value is in negative territory and will increase towards the 0 terminal value. If on the other hand, $b$ obeys condition (16) the costate value is in positive territory and will decline towards the 0 terminal state.

\section{Initial inventory in region $C$}

If the inventory falls in region $C$, ordering is carried out at maximum rate $(u=U)$ whilst the shadow price begins to drop. At time, $t=\tau_{2}$, the inventory crosses over to region $B$, where the shadow price is $\lambda_{2}=c \cdot \tau_{2}$ can be explicitly calculated again from (13) by setting the variable $z=b-c$ and solving for $\tau_{2}$ :

$$
\begin{aligned}
& \tau_{2}=T- \\
& \frac{1}{\left(4 a h^{2}\right)^{\frac{1}{3}}}\left(\ln \left(\left|\frac{b-\theta}{b-c-\theta}\right| \sqrt{\frac{(b-c)^{2}+(b-c) \theta+\theta^{2}}{b^{2}+b \theta+\theta^{2}}}\right)\right) \\
& +\frac{\sqrt{3}}{\left(4 a h^{2}\right)^{\frac{1}{3}}} \arctan \left(\frac{c \theta \sqrt{3}}{2 \theta^{2}+2 b(b-c)+\theta(2 b-c)}\right)
\end{aligned}
$$

\section{DISCUSSION}

In this work we have formulated an optimal inventory control problem and outlined its full solution when the demand is simultaneously both stock and price dependent and the order rate as well as the price are bounded. It was found that the maximum price, $b$, must fall outside the range 


$$
\left(\left(\frac{2 h}{a}\right)^{\frac{1}{3}}, c+\frac{3}{2}\left(\frac{2 h}{a}\right)^{\frac{1}{3}}\right) \text { if maximum profit is to be }
$$

feasible. This enables the inventory planner to set in advance a maximum price in terms of the three key parameters; the intrinsic demand growth rate, $a$, the unit holding cost, $h$, and the unit order cost, $c$ and set the pricing and ordering strategies according to which region the inventory happens to be in.

\section{References}

[1] H.B. Wolfe, "A model for control of style merchandise" Indiana Management Review, 9, pp. 69-82, 1968.

[2] R.C.Baker, T.L. Urban, "A deterministic inventory model with an inventory-level-dependent demand", Journal of the Operational Research Society, 39(9), pp. 823-831, 1988.
[3] T.L. Urban, "An inventory model with an inventory-level dependent demand rate and relaxed terminal conditions", Journal of the Operational Research Society, 43, pp. 721-724, 1992.

[4] T.L. Urban, "Inventory models with the demand rate dependnent on stock and shortage levels", International Journal of Production Economics, 40, pp. 21-28, 1995.

[5] Y. Gerchak, Y. Wang, "Periodic review inventory models with inventory-level dependent demand", Naval Research Logistics, 41, pp. 99-116, 1994.

[6] S. Jørgensen, P.M. Kort, "Optimal pricing and inventory policies: Cenrtralized and decentralized decision making", European Journal of Operational Research, 138, pp. 578-600, 2002.

[7] E. Khmelnitsky, Y. Gerchak, "Optimal Control Approach to Production System with Inventory-Level-Dependent Demand", IEEE Transactions of Automatic Control, 47(2), pp. 289-292, 2002.

[8] A.C. Chiang, Elements of Dynamic Optimization, McGraw-Hill, 1992.

[9] M.I. Kamien, N.L. Schwartz, "Sufficient Conditions in Optimal Control Theory", Journal of Economic Theory, 3, pp. 207-214, 1971.

\section{Creative Commons Attribution License 4.0 (Attribution 4.0 International, CC BY 4.0)}

This article is published under the terms of the Creative Commons Attribution License 4.0

https://creativecommons.org/licenses/by/4.0/deed.en_US 